\title{
Thermopower, entropy, and the Mott relation in HgSe:Fe
}

\author{
K. Ikeda, ${ }^{1}$ R. Fletcher, ${ }^{1}$ J. C. Maan, ${ }^{2}$ and J. Kossut ${ }^{3}$ \\ ${ }^{1}$ Physics Department, Queen's University, Kingston, Ontario, Canada K7L 3N6 \\ ${ }^{2}$ Research Institute for Materials, High Field Magnet Laboratory, University of Nijmegen, 6500 GL Nijmegen, The Netherlands \\ ${ }^{3}$ Institute of Physics, Polish Academy of Science, Aleja Lotników 32/46, 02-668 Warsaw, Poland
}

(Received 16 July 2001; published 11 December 2001)

\begin{abstract}
We have investigated the quantum oscillations in the diffusion thermopower of a $\mathrm{HgSe}$ crystal doped with about $1 \% \mathrm{Fe}$. The high concentration of Fe has provided sufficient attenuation of phonon-drag quantum oscillations to allow clear observation of oscillations in the diffusion thermopower of a degenerate semiconductor. At high magnetic fields the diffusion oscillations are well represented by the entropy per unit charge, though the measured amplitude is larger than expected by about 50\%. At low fields the oscillations shift in phase and agree with those predicted from the electronic relaxation time using the Mott relation.
\end{abstract}

DOI: 10.1103/PhysRevB.65.035201

PACS number(s): 72.20.Pa

\section{INTRODUCTION}

In the absence of phonons, thermopower is closely connected to carrier diffusion in a temperature gradient and therefore can, in principle, give direct access to fundamental thermodynamic properties of conductors. However, phonon interactions also give a contribution to thermopower, phonon drag, which typically masks the more fundamental diffusion contribution. In this paper we discuss results on diffusion thermopower on a specially chosen $\mathrm{HgSe}$ sample where the effect of phonons has been greatly reduced.

It has long been known, see, for example, Ioffe, ${ }^{1}$ that diffusion thermopower $S^{d}$ is related to the entropy of the charge carriers, $\mathcal{S}$ (defined here as the entropy per unit volume), for simple semiconductors. If electron-scattering effects can be ignored (in practice it is sufficient if the electron scattering is independent of energy) and with no magnetic field present, $S^{d}$ is equivalent to the electronic entropy per unit charge, i.e.,

$$
S^{d}=-\mathcal{S} / n|e|,
$$

where $n$ is the electron density and $|e|$ the magnitude of the electronic charge, the negative sign assumes that electrons rather than holes are the charge carriers. This result is known to be valid in the limits of degenerate and classical statistics of the carriers, but is probably valid for any degeneracy though we have seen no proof for the general case. It is also valid in both three and two dimensions and appears to remain true whatever the electron scattering in transverse, classically high magnetic fields. "Classically high magnetic fields" means that Landau quantization is ignored and this restriction arises because the result is based on the Boltzmann transport equation, which becomes invalid when Landau levels are dominant. Assuming sufficient symmetry in the $x y$ plane and with a transverse magnetic field $B$ along $z$, there are two independent components of the thermopower tensor, the thermopower $S_{x x}$ and the Nernst-Ettingshausen coefficient $S_{y x}$. The entropy result applies only to the former.

Obraztsov $^{2}$ showed that Eq. (1) is also valid for $S_{x x}^{d}$ in three dimensions (3D) in a magnetic field for the case of zero scattering of the electrons and applies to both the oscillatory part $\widetilde{S}_{x x}^{d}$ and the smooth background $\bar{S}_{x x}^{d}$. (Throughout we will use a bar and tilde to indicate monotonic and oscillatory components of the coefficients.) The Boltzmann equation cannot be applied to this situation regardless of the strength of the magnetic field. Later the result was extended to the case with electron scattering ${ }^{3}$ and was shown to remain valid but correction terms appear. We note that for negatively charged carriers the oscillations in $S_{x x}^{d}$ are in antiphase with the oscillations in the electronic density of states, which, in turn, are in phase with those in the resistivity $\widetilde{\rho}_{x x}$. The calculations have also been extended to $2 \mathrm{D}$ for zero and weak scattering ${ }^{4}$ but we will not consider this case further except to say that the entropy component has not been definitively observed there as yet.

Because Eq. (1) had been known for many years, it was not surprising that it should also hold when quantum effects dominate at high fields. Nevertheless, there is another result that has invariably been used in analyzing $S^{d}$ in degenerate systems, the Mott relation. This relates $S^{d}$ to the derivative of the conductivity with respect to electron energy. Although this was originally based on the Boltzmann equation, an analogous result has been shown to remain valid even in strong magnetic fields. ${ }^{5}$ In 3D this approach leads to the prediction that $\widetilde{S}_{x x}^{d}$ is shifted in phase by $\pi / 2$ compared to entropy oscillations, ${ }^{6}$ and also has a different field dependence. Although the situation is not completely clear, the theoretical work ${ }^{5,3}$ appears to suggest that the two contributions, Mott and entropy, should both exist, in general, at least at high fields when the scattering is not too strong. Only the entropy component would exist when there is no electronic scattering and the Mott component can be considered to be correction to allow for the effects of electronic scattering.

Given the phase shift and generally different amplitudes between the Mott and entropy results in 3D, one might expect that it would be a straightforward matter to distinguish the two contributions experimentally, but this has not proved to be the case. The main reason is that there is another contribution to thermopower, the phonon $\operatorname{drag} S^{g}$ due to nonequilibrium phonons in the temperature gradient pumping momentum into the electron system via electron-phonon col- 
lisions. Classically $S_{x x}^{g}$ is predicted ${ }^{7}$ to be independent of $B$ but in reality the electron-phonon relaxation time also oscillates in the presence of Landau levels. There are no theoretical results dealing with the resulting quantum oscillations in $S_{x x}^{g}$, but for negative carriers they are expected to be in antiphase with the oscillations in the density of states and this has experimentally been seen to be the case when $S_{x x}^{g}$ is completely dominant. ${ }^{6}$ This means that they are not easily distinguishable from entropy oscillations in $S_{x x}^{d}$.

Although this is a long-standing problem, as far as we are aware there are only two previous papers concerned with experimentally investigating the oscillations in the thermopower of 3D semiconductors. Schroder and Landwehr ${ }^{8}$ made an early study of $\widetilde{S}_{x x}$ in HgSe specifically to check the Obraztsov entropy result. They found that the oscillations were in antiphase with $\widetilde{\rho}_{x x}$ as predicted and, with a suitable choice of various parameters, the field dependence of the oscillations could also be made to agree with the Obraztsov result. However, the absolute magnitude of $\widetilde{S}_{x x}^{d}$ was not measured and the presence of $\widetilde{S}_{x x}^{g}$ was not appreciated at the time. Thus there remains some doubt as to the significance of those measurements.

More recently, Tieke et al. ${ }^{6}$ made detailed measurements on both components of $S_{i j}$ and of the resistivity $\rho_{i j}$ for $\mathrm{HgSe}$ doped with $0.03 \% \mathrm{Fe}$. It was found that $S^{g}$ was very large at low temperatures. The oscillatory part $\tilde{S}_{x x}^{g}$ was probably dominant up to about $20 \mathrm{~K}$ and could not be ignored up to temperatures of order $50 \mathrm{~K}$ so that $\widetilde{S}_{x x}^{d}$ could not be evaluated. Interestingly, $S_{y x}^{g}$ is predicted to be zero ${ }^{7}$ and it was found that the experimental results on $S_{y x}$, which were then presumably only $S_{y x}^{d}$, could be accurately reproduced by calculations based on the Mott relation at all fields and temperatures. Recall that there is no entropy component in $S_{y x}^{d}$.

Clearly the key to the experimental problem of observing $\widetilde{S}_{x x}^{d}$ is to reduce $S^{g}$ and thereby $\widetilde{S}_{x x}^{g}$. In general, this can be done by adding impurities that strongly scatter phonons. Unfortunately, this also usually reduces the electron mobility very rapidly so that quantum oscillations are no longer visible. Tieke et al. ${ }^{6}$ chose the system $\mathrm{HgSe}+0.03 \%$ Fe because Fe impurity can be added to $\mathrm{HgSe}$ to give a high electron concentration, and thus many oscillations, while retaining a high mobility. However, it is known that the $\mathrm{HgSeFe}$ system is unusual in that considerably more Fe may be introduced, up to at least $1 \%$, with relatively little effect on either electron mobility or density (for a recent review, see Ref. 9). Such a large $\mathrm{Fe}$ content has also been shown ${ }^{10}$ to give a strong decrease in $S^{g}$.

Owing to these attractive properties, we have investigated the thermopower of $\mathrm{HgSe}$ sample doped with about $1 \% \mathrm{Fe}$ and the present paper reports our results. Briefly, the expected reduction of $S^{g}$ was observed, and we find strong evidence that both contributions to $\widetilde{S}_{x x}^{d}$ exist in magnetic fields, with the entropy and Mott results being dominant at high and low fields, respectively.

\section{THEORY}

This section introduces the main theoretical results that are required to analyze the data. Because we are interested in the relative phases of $\widetilde{S}_{x x}^{d}$ and $\widetilde{\rho}_{x x}$, it was necessary to experimentally investigate the latter in some detail. Resistivity oscillations reflect the oscillations in the electronic density of states at the Fermi energy $\varepsilon_{F}$ via changes in the relaxation time $\tau$ of the electrons. For degenerate electrons with spherical energy surfaces and elastic scattering, it is predicted ${ }^{11}$ that

$$
\begin{aligned}
\rho_{x x}= & \bar{\rho}_{x x}+\widetilde{\rho}_{x x}=\rho_{0}\left[1+\frac{5}{2}\left(\frac{B}{2 f}\right)^{1 / 2} \sum_{r=1}^{\infty} \frac{(-1)^{r}}{r^{1 / 2}} D(r X)\right. \\
& \left.\times \cos \left(\frac{2 \pi f}{B} r-\frac{\pi}{4}\right) \exp \left(-\frac{\gamma T_{D}}{B} r\right)\right],
\end{aligned}
$$

where $\rho_{0}$ is the resistivity at zero field, $D(X)=X / \sinh X$ is a thermal damping factor with $X=2 \pi^{2} k_{B}^{2} T / \hbar \omega_{c}$ and $\omega_{c}$ being the cyclotron frequency, and $T_{D}$ is the Dingle temperature that accounts for collision broadening of the Landau levels. The ratio $f / B=\varepsilon_{F} / \hbar \omega_{c}$. We have dropped a small extra oscillatory term, which is negligible in this work, and also ignored a spin-splitting term that will be introduced later.

Although the oscillations in $\rho_{y x}$ are very small and have not attracted much experimental attention (the most comprehensive set of data seem to be those of Mani, Anderson, and Johnson ${ }^{12}$ ), they are required to give a complete picture of the thermopower oscillations. Equations for the Hall conductivity $\sigma_{x y}$ have been given by Horton, ${ }^{13}$ Guseva and Zyryanov, ${ }^{14}$ and Zyryanov and Kuleyev ${ }^{15}$ (the last two papers give a result only for the fundamental component at zero temperature). Horton gives

$$
\begin{aligned}
\sigma_{x y}= & \bar{\sigma}_{x y}+\widetilde{\sigma}_{x y}=-\frac{n|e|}{B}+\frac{n|e|}{B\left(1+\beta^{2}\right)} \\
& \times\left[1+\frac{7 \sqrt{2}}{8}\left(\frac{B}{2 f}\right)^{1 / 2} \sum_{r=1}^{\infty} \frac{(-1)^{r}}{r^{1 / 2}} D(r X) \cos \left(\frac{2 \pi f}{B} r\right.\right. \\
& \left.\left.-\frac{\pi}{4}\right) \exp \left(-\frac{\gamma T_{D}}{B} r\right)\right],
\end{aligned}
$$

where $\beta=\omega_{c} \tau$. We use $\rho_{y x}=\sigma_{x y} /\left(\sigma_{x y}^{2}+\sigma_{x x}^{2}\right)$ where $\sigma_{x x}$ is obtained from Eq. (2) (using a similar relation between $\sigma_{x x}$ and $\left.\rho_{x x}\right)$ and find for $\beta^{2} \gg 1$

$$
\begin{aligned}
\rho_{y x}= & \bar{\rho}_{y x}+\widetilde{\rho}_{y x}=-\frac{B}{n|e|}\left[1-\frac{3}{2}\left(\frac{B}{2 f}\right)^{1 / 2} \frac{1}{\beta^{2}} \sum_{r=1}^{\infty} \frac{(-1)^{r}}{r^{1 / 2}}\right. \\
& \left.\times D(r X) \cos \left(\frac{2 \pi f}{B} r-\frac{\pi}{4}\right) \exp \left(-\frac{\gamma T_{D}}{B} r\right)\right] .
\end{aligned}
$$

It is possible that these results may not be complete for the case of $\mathrm{HgSe}: \mathrm{Fe}$ because it has been postulated that for sufficient Fe content (as here) the Fermi energy $\varepsilon_{F}$ is pinned in the conduction band by the Fe impurity state. ${ }^{16}$ If this is correct, and if it remains valid under dynamic conditions at low temperature, then the number of electrons will oscillate with changing magnetic field and will result in another contribution to $\widetilde{\rho}_{y x}$. 
The thermopower is defined by $E_{i}=S_{i j} \nabla T_{j}$, where $\bar{E}$ is the measured electric field and $\boldsymbol{\nabla} T$ is the temperature gradient. The situation is complicated by the fact that the present sample has a relatively large contribution to the thermal conductivity from the electrons, $\lambda_{i j}^{e}$, which gives a finite transverse temperature gradient $\nabla T_{y}$. With no transverse heat current, $\quad \nabla T_{y} / \nabla T_{x}=-\beta \lambda_{0}^{e} /\left[\lambda^{g}\left(1+\beta^{2}\right)+\lambda_{0}^{e}\right] \approx-\lambda_{0}^{e} / \beta \lambda^{g}$, assuming free-electron results and elastic scattering for $\lambda_{i j}$, with $\lambda_{0}^{e}$ being the electronic thermal conductivity at zero field. This gives a measured thermopower

$$
S_{x x}^{m} \approx S_{x x}+S_{y x}\left(\lambda_{0}^{e} / \beta \lambda^{g}\right),
$$

which we shall use below. The thermal conductivity at zero field is $\lambda^{g}+\lambda_{0}^{e}$. When $\beta^{2} \gg 1$ we expect a measured thermal conductivity of $\approx \lambda^{g}+\left(\lambda_{0}^{e} / \beta^{2}\right)\left[1+\left(\lambda_{0}^{e} / \lambda^{g}\right)\right]$, which approaches $\lambda^{g}$ as $\sim 1 / B^{2}$ for $\beta \gg 1$.

The semiclassical (Mott) results for the diffusion components $\bar{S}_{i j}^{d}$ with elastic scattering and degenerate electrons $\operatorname{are}^{6,17}$

$$
\begin{gathered}
\bar{S}_{x x}^{d}=-\frac{\pi^{2} k_{B}^{2} T}{3|e| \varepsilon_{F}}\left[\frac{3}{2}+\frac{p}{1+\beta^{2}}\right], \\
\bar{S}_{y x}^{d}=-\frac{\pi^{2} k_{B}^{2} T}{3|e| \varepsilon_{F}}\left[\frac{p \beta}{1+\beta^{2}}\right],
\end{gathered}
$$

where $p=(\partial \ln \tau / \partial \ln \varepsilon)_{\varepsilon_{F}}$. Using Eq. (5) we see that, whatever the value of $\lambda_{0}^{e} / \lambda^{g}$, the limiting high-field result for $\bar{S}_{x x}^{d}$ reduces to $-\pi^{2} k_{B}^{2} T / 2|e| \varepsilon_{F}$, which is the entropy per unit charge, i.e., Eq. (1), though $\lambda_{x x}^{e}$ might have some effect on how $S_{x x}^{M}$ makes the transition from low to high field.

There is an extension of the Mott result to oscillatory components, which are found to be ${ }^{6}$

$$
\begin{gathered}
\widetilde{S}_{x x}^{d}=-\frac{\alpha}{1+\beta^{2}}\left(\frac{\widetilde{\rho}_{x x}}{\bar{\rho}_{x x}}+\beta^{2} \frac{\widetilde{\rho}_{y x}}{\bar{\rho}_{y x}}\right), \\
\widetilde{S}_{y x}^{d}=-\frac{\alpha \beta}{1+\beta^{2}}\left(\frac{\widetilde{\rho}_{x x}}{\bar{\rho}_{x x}}\right),
\end{gathered}
$$

where $\quad \alpha=i\left(\pi k_{B} /|e|\right) D^{\prime}(r X) / D(r X) \quad$ and $\quad D^{\prime}(r X)=[1$ $-r X \operatorname{coth}(r X)] / \sinh (r X)$ is the thermal damping factor for thermopower oscillations and $i$ signifies that there is a phase shift of $\pi / 2$ between $\widetilde{\rho}_{i j}$ and $\widetilde{S}_{i j}^{d}$. Although these last results were first derived using the Boltzmann equation, theoretical results by Smrčka and Streda ${ }^{5}$ suggest that they are more general. In support of this view we note that Eq. (9) has been shown to be accurately obeyed in $\mathrm{HgSe}: \mathrm{Fe}$ under similar conditions of temperature and field as used here. ${ }^{6}$ [Note that the signs of $\widetilde{S}_{x x}^{d}$ and $\widetilde{S}_{y x}^{d}$ given in Eqs. (6a) and (6b) of Ref. 6 should both have been negative as above.] We shall refer to the measured thermopower from this source as the Mott contribution $\widetilde{S}_{x x}^{M}$, which from Eqs. (5), (8), and (9) is given by

$$
\widetilde{S}_{x x}^{M}=-\frac{\alpha}{1+\beta^{2}}\left(\frac{\widetilde{\rho}_{x x}}{\bar{\rho}_{x x}}\left[1+\frac{\lambda_{0}^{e}}{\lambda^{g}}\right]+\beta^{2} \frac{\widetilde{\rho}_{y x}}{\bar{\rho}_{y x}}\right) .
$$

Finally, using Eqs. (2) and (4), $\widetilde{S}_{x x}^{M}$ is predicted to be

$$
\begin{aligned}
\widetilde{S}_{x x}^{M}= & \frac{\pi k_{B}}{|e|} \frac{1+\left(5 \lambda_{0}^{e} / 2 \lambda^{g}\right)}{1+\beta^{2}}\left(\frac{B}{2 f}\right)^{1 / 2} \sum_{r=1}^{\infty} \frac{(-1)^{r}}{r^{1 / 2}} D^{\prime}(r X) \\
& \times \sin \left(\frac{2 \pi f}{B} r-\frac{\pi}{4}\right) \exp \left(-\frac{\gamma T_{D}}{B}\right) .
\end{aligned}
$$

Obraztsov $^{2}$ studied the case of no electronic scattering where Eq. (11) gives a zero result. He found the following, which we refer to as $S_{x x}^{O}$ (there is no contribution to $S_{y x}$ ),

$$
\begin{aligned}
S_{x x}^{O}= & \bar{S}_{x x}^{d}+\widetilde{S}_{x x}^{d}=-\frac{\pi^{2} k_{B}^{2} T}{2|e| \varepsilon_{F}}+\frac{3 k_{B}}{2|e|}\left(\frac{B}{2 f}\right)^{3 / 2} \sum_{r=1}^{\infty} \frac{(-1)^{r}}{r^{3 / 2}} \\
& \times D^{\prime}(r X) \cos \left(\frac{2 \pi f}{B} r-\frac{\pi}{4}\right)
\end{aligned}
$$

This equation is equivalent to the entropy of the electrons per unit charge. We assume that when the Landau levels are broadened by scattering, a collision-broadening term, $\exp \left(-\gamma r T_{D} / B\right)$, must be included in the oscillatory part. Because $D^{\prime}(X)$ is a negative quantity, $\widetilde{S}_{x x}^{O}$ as predicted by Eq. (12) is in antiphase with $\widetilde{\rho}_{x x}$ as given by Eq. (2). Also $\widetilde{S}_{x x}^{O}$ is distinguishable from $\widetilde{S}_{x x}^{M}$ given by Eq. (10), which is $\pi / 2$ out of phase. Finally note that $\bar{S}_{x x}^{d}$ is the same as that given by Eq. (6) when $\beta \rightarrow \infty$.

\section{EXPERIMENTAL TECHNIQUES AND RESULTS}

A sample was cut from a single crystal of $\mathrm{HgSe}$ doped with nominally $1 \% \mathrm{Fe}$ using a diamond impregnated wire saw and lapped to a rectangular shape about $8 \times 2.3$ $\times 0.9 \mathrm{~mm}^{3}$. This was a rather small sample that should help to minimize any problems with inhomogeneity. It was polished with $1 \% \mathrm{Br}$ in methanol and, after cleaning with benzene, given a $10 \mathrm{~min}$ dip at $40^{\circ} \mathrm{C}$ in an acid etch recommended by Warekois et al. ${ }^{18}$ Following this treatment the sample was readily soldered with indium. Six $25-\mu \mathrm{m}-$ diameter gold leads were attached as current and potential probes, and these in turn were soldered to $50-\mu \mathrm{m}$ manganin leads to provide thermal isolation. The indium contacts all had very low resistance. Finally the sample was indium soldered at one end to the cold sink. The remaining setup was similar to that used by Tieke et al. ${ }^{6}$

The small sample combined with the finite width of the indium contacts means that the resistivity could be in error by about $15 \%$. However, we will only need the ratio of $\widetilde{\rho}_{x x} / \rho_{0}$ [see Eq. (2)], which we would expect to be much more accurate unless the effective contact spacing is field dependent. The thermopower needs the extra measurement of the length between the thermometer probes. This raises the overall uncertainty to about $20-25 \%$. In the analysis we will use the high-field limit of the smooth part of the thermopower to normalize the data so that the resulting error on the absolute magnitude should be much lower.

The electron density was found from Hall data to be 5.2 $\times 10^{24} \mathrm{~m}^{-3}( \pm 1 \%)$ and independent of temperature. The mo- 


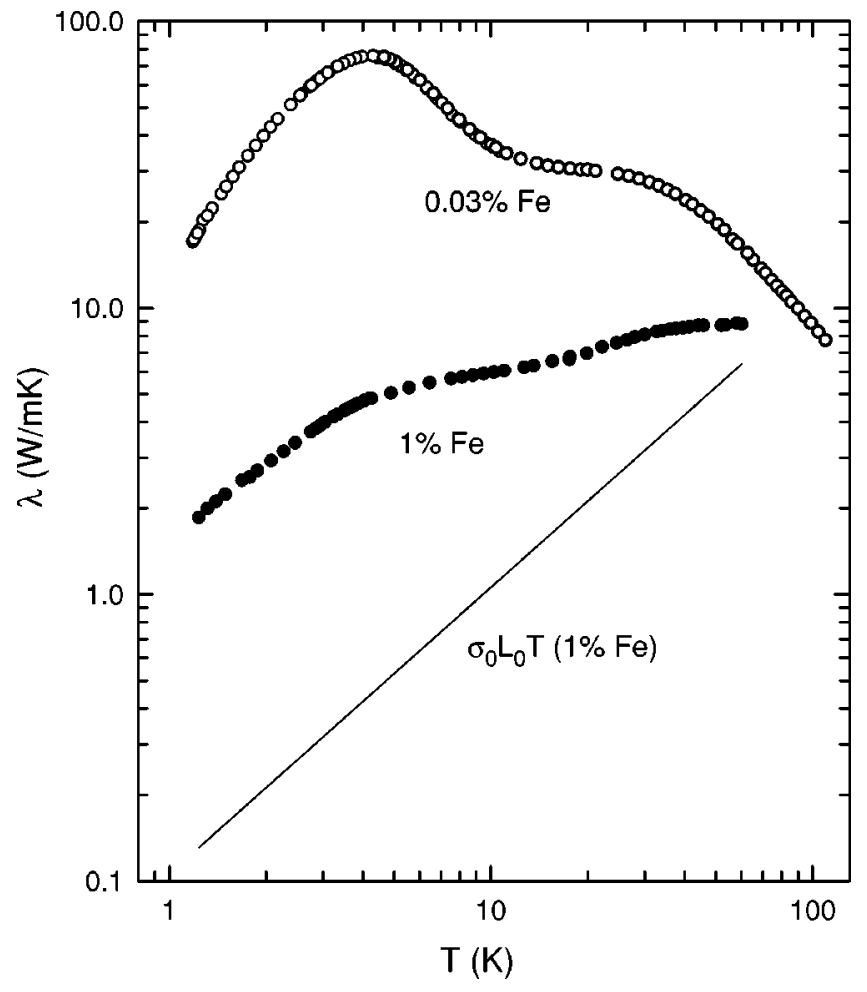

FIG. 1. Thermal conductivity $\lambda$ at zero field as a function of temperature. The closed symbols are for the present sample and the open symbols are from the earlier work of Tieke et al. ${ }^{6}$ The line is an upper estimate for electronic thermal conductivity calculated using the measured conductivity at $4.2 \mathrm{~K}, \sigma_{0}$, and the Sommerfeld value of the Lorenz number $L_{0}$.

bility was somewhat temperature dependent ranging from $5.2 \mathrm{~m}^{2} / \mathrm{V} \mathrm{s}$ at ${ }^{4} \mathrm{He}$ temperatures to $4.0 \mathrm{~m}^{2} / \mathrm{V} \mathrm{s}$ at $42 \mathrm{~K}$. Figure 1 shows data on the thermal conductivity $\lambda$ of the present sample, together with data from the study by Tieke et al. ${ }^{6}$ on a HgSe sample with nominally $0.03 \% \mathrm{Fe}$. The extra Fe has greatly increased phonon scattering in the present sample, thereby strongly decreasing $\lambda^{g}$. As a result the electronic part $\lambda_{0}^{e}$ is relatively much larger in the present sample. The line gives an estimate of $\lambda_{0}^{e}$ assuming the validity of the Wiedemann-Franz law, but it is known that the Lorenz number begins to drop rapidly in the region of interest. ${ }^{19}$ We can obtain a better estimate of $\lambda_{0}^{e} / \lambda^{g}$ using the magnetic-field dependence of the measured $\lambda$. Over the range $20-40 \mathrm{~K}$ the ratio is only weakly temperature dependent and has an average value of about 0.4 .

The thermopower at zero field for both samples is given in Fig. 2. The straight line is the estimated diffusion thermopower $S^{d}$ (assuming that the high-temperature asymptote gives a good guide) and is essentially the same for both samples. We attribute deviations from this line to $S^{g}$. The strong reduction seen in $\lambda$ is also seen in $S^{g}$. In the present sample $S^{g}$ becomes very small above about $20 \mathrm{~K}$. Although there are no predictions concerning the magnitude of $\widetilde{S}_{x x}^{g}$, a consideration of their physical origin suggests $\tilde{S}_{x x}^{g} / \bar{S}_{x x}^{g}$ $\leqslant \tilde{\rho}_{x x} / \bar{\rho}_{x x}$. It follows that $\widetilde{S}_{x x}^{g}$ should be negligible above $\sim 20 \mathrm{~K}$ so the data on $\widetilde{S}_{x x}$ should be dominated by $\widetilde{S}_{x x}^{d}$ in this

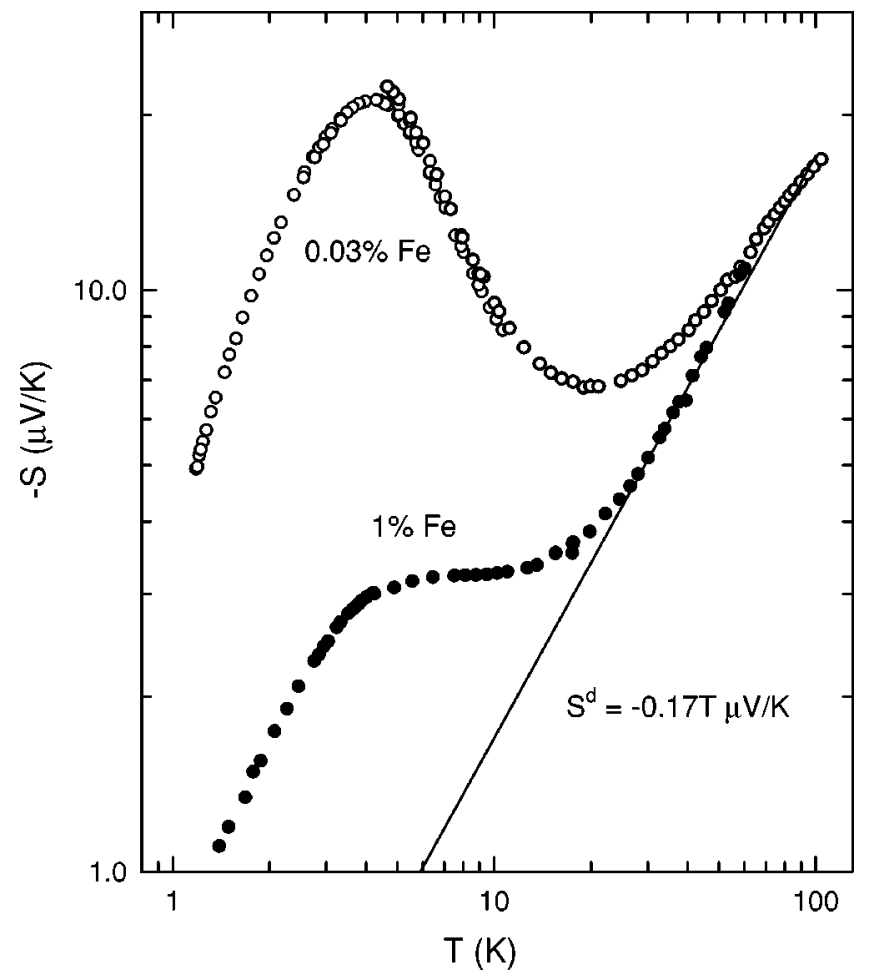

FIG. 2. Thermopower $S$ at zero field as a function of temperature. The symbols are defined in Fig. 1. The line is the estimated diffusion component $S^{d}$.

region; we shall assume this in what follows.

To obtain the field dependence of both $\rho_{x x}$ and $S_{x x}$ it was necessary to take field sweeps with both $\pm B$ and evaluate the even parts, e.g., $S_{x x}=\left[S_{x x}(+B)+S_{x x}(-B)\right] / 2$. Similarly we used the odd part in evaluating $\rho_{y x}$. Measurements were made only during sweeps with increasing field to minimize any hysteresis problems with the superconducting magnet. Finally, all data for both $\rho_{x x}$ and $S_{x x}$ were taken with the same pair of voltage probes on the sample because we require the relative phases of the oscillations in each of these coefficients and this arrangement minimizes, or even eliminates, inhomogeneity effects.

We will not show examples of raw data for $\rho_{x x}$ because they are very similar to those given by Tieke et al. ${ }^{6}$ The current in the sample was limited to $\leqslant 5 \mathrm{~mA}$ to avoid any temperature gradient (we can detect about $1 \mathrm{mK}$ ) and unwanted admixture of a thermopower signal.

Figure 3 shows $S_{x x}$ (which we now take to be $S_{x x}^{d}$ ) as a function of magnetic field at various fixed temperatures from about 22-44 K. The upper temperature limit of the measurements was dictated by the thermometers becoming too insensitive. At low fields where $S_{x x}$ varies rapidly, the thermal conductivity also has a strong magnetic-field dependence due to the presence of $\lambda_{i j}^{e}$. This leads to the temperature and temperature gradient of the sample being functions of field. Our interest is primarily in the data at high fields where these corrections decrease as $1 / B^{2}$ and become very small. Hence we have not corrected the results for these effects at low fields. With this proviso in mind, the smooth backgrounds of the data are consistent with the field dependence predicted by 


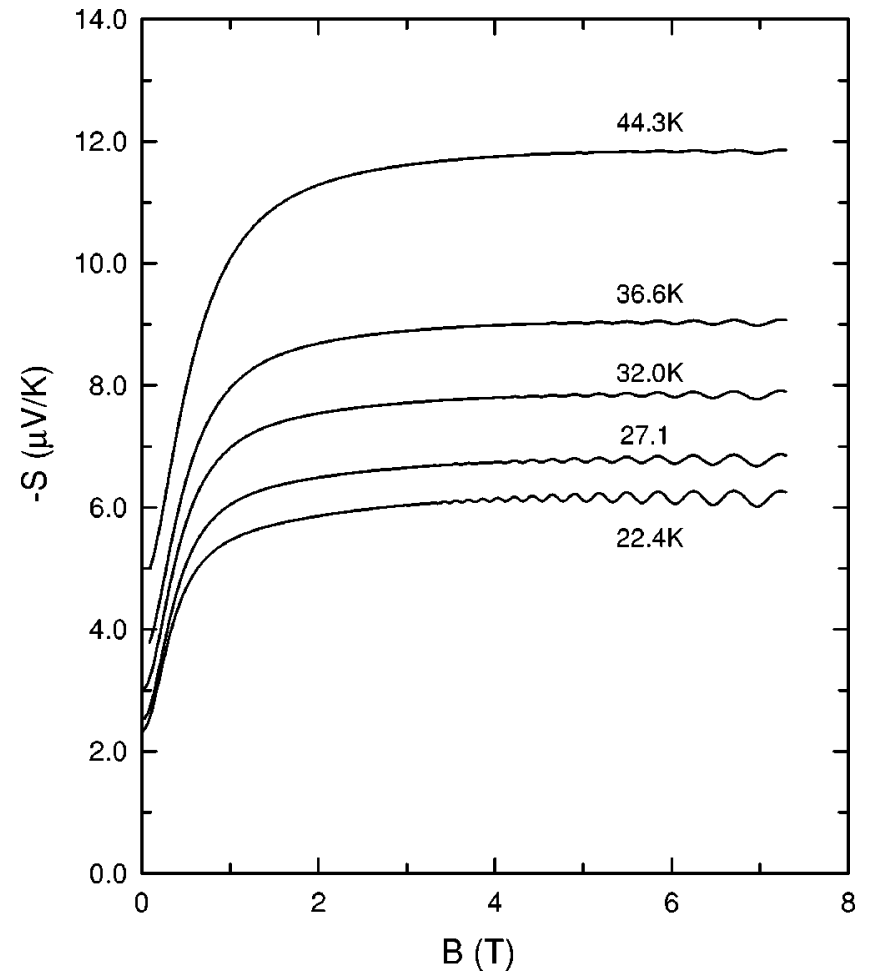

FIG. 3. Thermopower $S_{x x}$ as a function of field at the various temperatures used in these experiments. These data are believed to be almost totally $S_{x x}^{d}$.

Eq. (6) and are similar to those seen by Tieke et al. ${ }^{6}$ However, it is noticeable that the oscillations in the present data are always smaller than those reported in this previous work at similar temperatures. This is in accord with the fact that we expect $\widetilde{S}_{x x}^{g}$ to be significant in the earlier sample. The saturation values of $\bar{S}_{x x}^{d}$ will be mentioned again in the following section.

\section{ANALYSIS}

The goal of the analysis is to compare the experimental data on $\widetilde{S}_{x x}^{d}$ in Fig. 3 with Eqs. (10) and (12). We first analyze $\widetilde{\rho}_{x x}$ to give the reference frequencies and phases that we need to distinguish the two contributions to the thermopower.

The de Haas-Shubnikov oscillations at the lowest temperature of $7.6 \mathrm{~K}$ have more than one frequency component (see the data of Tieke et ll $^{6}$ ). Fourier analysis shows that there are two close frequencies near $91 \mathrm{~T}$ of approximately equal amplitude, and also second and third harmonics of these frequencies. To simplify the analysis we first removed the harmonics by filtering using a pass band of 50-150 T. This procedure also removes the smooth backgrounds though it was found to be better to first eliminate most of the background by fitting to a low-power polynomial. The results are shown in Fig. 4 as a function of $1 / B$ where it is seen that there is now a simple beat pattern arising from the two close frequencies.

These two frequencies do not have their origin in a nonspherical Fermi surface, but rather in the lack of inversion

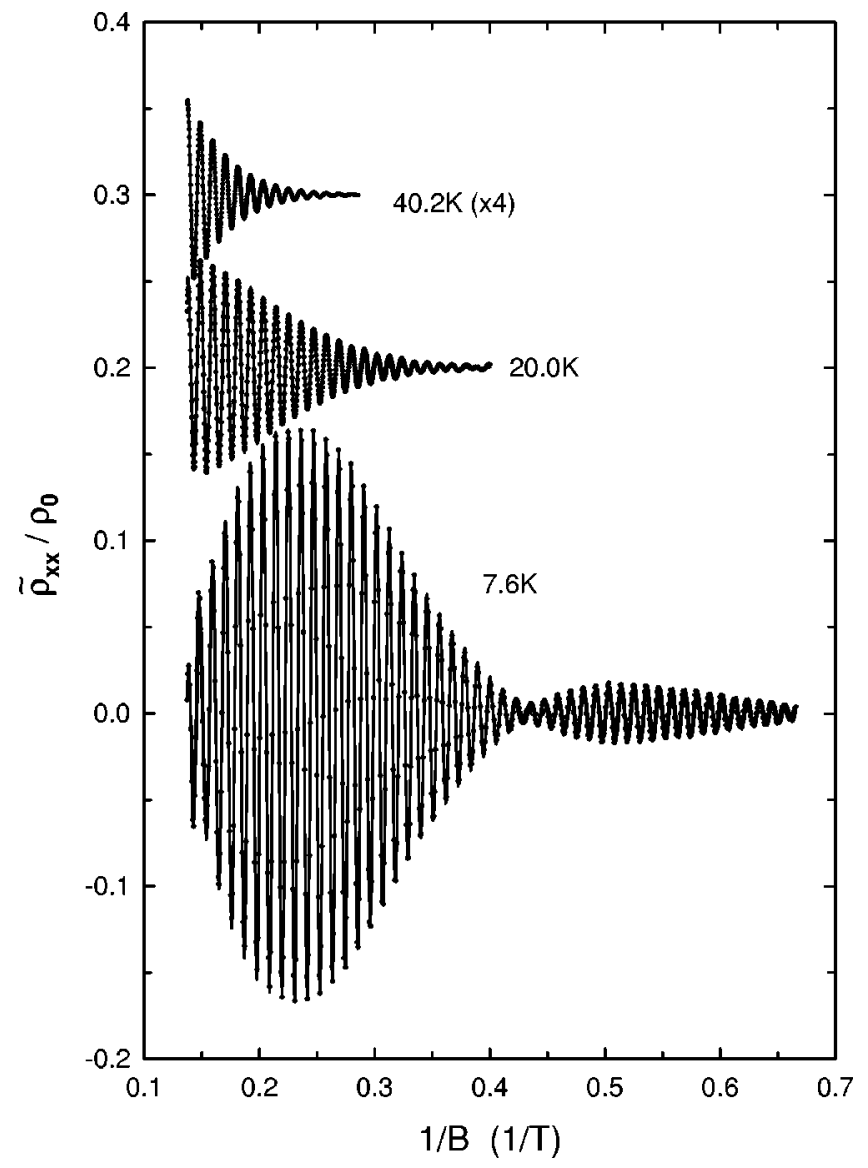

FIG. 4. Examples of the resistivity oscillations $\widetilde{\rho}_{x x}$ as a function of inverse field. Note the scale change for the data at $40.2 \mathrm{~K}$. The dots represent $25 \%$ of the measured data points and the curves through the points were fitted as described in the text.

symmetry of the crystal lattice, which causes an energy difference between electrons of opposite spin. ${ }^{20}$ This has been extensively studied in previous work (see Ref. 21 and references therein). If we take the components from opposite spin to have the same amplitude and assume that the spin-up and -down components simply act in parallel, we can use Eq. (2) to write the fundamental components as

$$
\begin{aligned}
\frac{\tilde{\rho}_{x x}}{\rho_{0}}= & -\frac{5}{4}\left(\frac{B}{2 f_{\mathrm{avg}}}\right)^{1 / 2} D(X)\left[\cos \left(\frac{2 \pi f_{1}}{B}-\phi_{1}\right)\right. \\
& \left.+\cos \left(\frac{2 \pi f_{2}}{B}-\phi_{2}\right)\right] \exp \left(-\frac{\gamma T_{D}}{B}\right) \\
= & -\frac{5}{2}\left(\frac{B}{2 f_{\mathrm{avg}}}\right)^{1 / 2} D(X) \cos \left(\frac{2 \pi f_{\mathrm{avg}}}{B}-\phi_{\mathrm{avg}}\right) \\
& \times \cos \left(\frac{2 \pi \Delta f}{B}-\Delta \phi\right) \exp \left(-\frac{\gamma T_{D}}{B}\right),
\end{aligned}
$$

where $f_{\text {avg }}=\left(f_{1}+f_{2}\right) / 2, \Delta f=\left(f_{1}-f_{2}\right) / 2$, etc. and we have made no assumptions about the values of $\phi_{1}$ and $\phi_{2}$.

As Fig. 4 shows, Eq. (13) gives an excellent fit to the experimental data at $7.6 \mathrm{~K}$ using as variables $f_{\text {avg }}, \Delta f, \phi_{\text {avg }}$, $\Delta \phi, T_{D}$, and the amplitude, and fixing $m^{*}=0.065 m_{e}$. This 
value of $m^{*}$ was used by Tieke et al. ${ }^{6}$ and is consistent with that of Miller and Reifenberger ${ }^{21}$ who give $(0.067$ $\pm 0.01) m_{e}$ for samples with similar Fe content and electron density. The second form of the equation was much easier to fit to experimental data than the first.

As one might expect, the value of the amplitude depends somewhat on the value of $m^{*}$ assumed, though the dependence is not very strong at $7.6 \mathrm{~K}$ because the damping function $D(X)$ becomes independent of $X$ for small $X$. We obtained an amplitude range of $2.95 \pm 0.10$ for a range of $m^{*}$ $=(0.065 \mp 0.02) m_{e}$. (Note that the amplitude is a relative measurement in terms of $\rho_{0}$ and so the size of the potential contacts should not be relevant provided their effective separation is not a function of the field.) It is difficult to put a precise uncertainty on the absolute amplitude from all other sources, but it is expected to be less than $10 \%$. This result is in good agreement with the predicted value of $\frac{5}{2}$. Although many papers exist dealing with the various terms in Eq. (2), we know of no previous measurement of the absolute amplitude of $\widetilde{\rho}_{x x}$. We found $T_{D}=(1.8 \pm 0.1) \mathrm{K}$ for the same range of $m^{*}$, which is also in good agreement with that of Miller and Reifenberger for a similar sample. ${ }^{21}$

The oscillations in $\rho_{x x}$ at higher temperatures were also separated from the background and filtered in the same way. The resulting data are also shown in Fig. 4. Because there are fewer oscillations, the Fourier transforms are broadened and do not indicate whether the two frequencies are still present. Nevertheless, these data cannot be satisfactorily fitted to a single frequency as in Eq. (2), but Eq. (13) still provides an excellent representation using the same values of $f_{\text {avg }}, \Delta f$, and $T_{D}$ as found above. The phases, $\phi_{\text {avg }}$ and $\Delta \phi$, were left as adjustable constants. This was essential in the case of the latter, which smoothly increases by about 1 rad over the range $7-42 \mathrm{~K}$. On the other hand $\phi_{\text {avg }}$ is constant within $\pm 0.1 \mathrm{rad}$. The fact that $\Delta \phi$ varies with temperature has been noted previously ${ }^{22}$ and attributed to interactions between the electron spin and crystal magnetization. The average absolute amplitude for all the data, except those at $7.6 \mathrm{~K}$ treated above, is $2.56 \pm 0.16$ with $m^{*}=0.065 m_{e}$, where the error is the statistical uncertainty. This agrees well with the predicted value of $\frac{5}{2}$ but at these higher temperatures the value of the amplitude depends sensitively on $m^{*}$. A change in $m^{*}$ of $\pm 0.002 m_{e}$, a reasonable range given in previous work, gives a change in amplitude of $\pm 13 \%$. Although small errors in $T$ are more serious at these higher temperatures, the consistency in the data suggests that the additional error in the absolute amplitude is probably still no more than about $10 \%$.

At this point the data on $\widetilde{\rho}_{x x}$ have been completely parametrized and we are now able to consider $\widetilde{S}_{x x}^{d}$. We removed most of the smooth background by fitting to Eq. (6), and filtered the remainder with the same passband as for $\tilde{\rho}_{x x}$. Some of the results are shown in Fig. 5. A close examination of the oscillations shows that $-\widetilde{S}_{x x}^{d}$ is almost in phase with $\tilde{\rho}_{x x}$ at high fields but the phase smoothly changes until it is shifted by almost $\pi / 2$ at the lowest fields; this is particularly noticeable in the case of the data at $22.4 \mathrm{~K}$, which extend to the lowest fields. One might question whether this could be due to sample inhomogeneities affecting resistivity and ther-

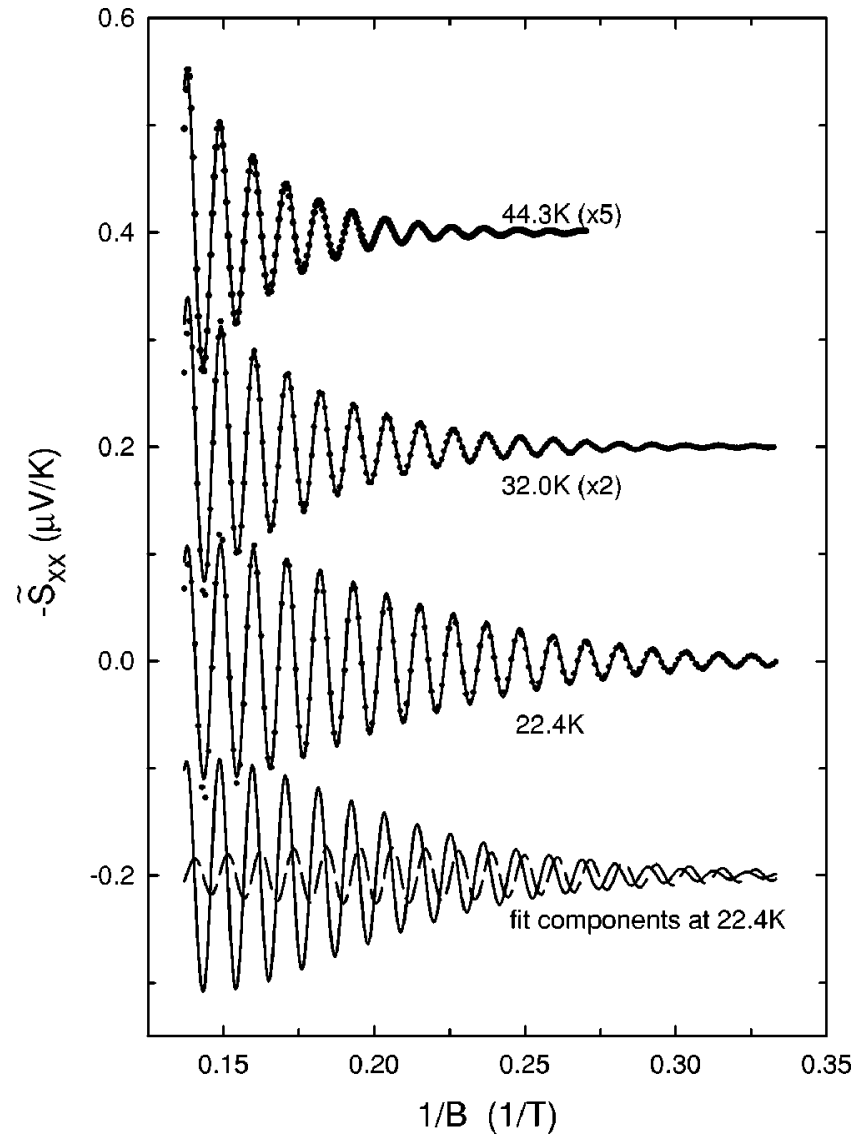

FIG. 5. Examples of the thermopower oscillations $\widetilde{S}_{x x}$ as a function of inverse field. Note the scale changes and the vertical offsets. $25 \%$ of the measured points are shown as dots and the lines are calculated as explained in the text. The lowest curves show the fitted components at $22.4 \mathrm{~K}$ with the solid line being the entropy part (dominant at high fields) and the dashed line the Mott part (dominant at low fields).

mopower slightly differently even though the same sample contacts were used for both. Against this it is found that at about $7 \mathrm{~K}$ where $\widetilde{S}_{x x}^{g}$ dominates, the oscillations remain in phase much more accurately over the whole field range.

We have analyzed the data quantitatively as follows. The entropy contribution will also have two frequency components and so we write the fundamental of Eq. (12) in the form of Eq. (13), i.e.,

$$
\begin{aligned}
S_{x x}^{O}= & -\frac{3 k_{B}}{2|e|}\left(\frac{B}{2 f}\right)^{3 / 2} D^{\prime}(X) \cos \left(\frac{2 \pi f_{\text {avg }}}{B}-\phi_{\text {avg }}\right) \\
& \times \cos \left(\frac{2 \pi \Delta f}{B}-\Delta \phi\right) \exp \left(-\frac{\gamma T_{D}}{B}\right) .
\end{aligned}
$$

Again allowing for the two frequencies in the Mott result (10) we obtain

$$
\begin{aligned}
\widetilde{S}_{x x}^{M}= & -\frac{\pi k_{B}}{|e|} \frac{\left(1+5 \lambda_{0}^{e} / 2 \lambda^{g}\right)}{1+\beta^{2}}\left(\frac{B}{2 f}\right)^{1 / 2} D^{\prime}(X) \sin \left(\frac{2 \pi f_{\mathrm{avg}}}{B}\right. \\
& \left.-\phi_{\mathrm{avg}}\right) \cos \left(\frac{2 \pi \Delta f}{B}-\Delta \phi\right) \exp \left(-\frac{\gamma T_{D}}{B}\right) .
\end{aligned}
$$




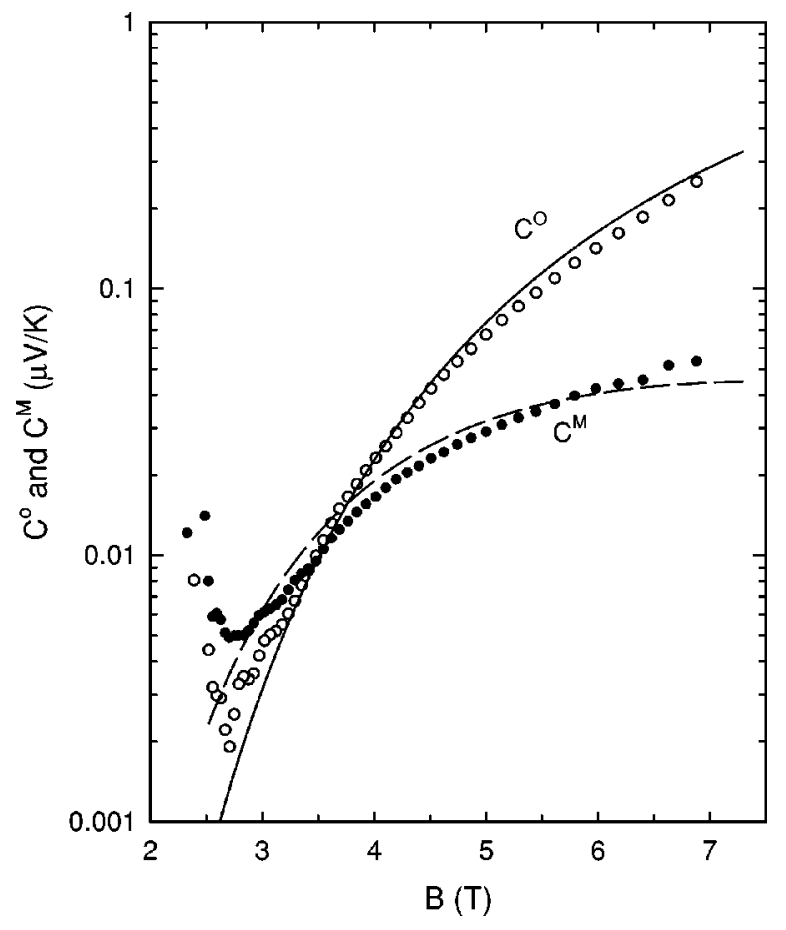

FIG. 6. Local amplitudes of the components of $\widetilde{S}_{x x}^{d}$ at $22.4 \mathrm{~K}$ that are in phase $\left(C^{O}\right)$ and $\pi / 2$ out of phase $\left(C^{M}\right)$ with $\tilde{\rho}_{x x}$ as a function of field. The solid lines are the expected variations with the overall absolute amplitudes adjusted to give the best fits.

We first show how the two components contribute to the measured $\widetilde{S}_{x x}$ as a function of $B$. The separation was made by fitting successive single periods of the oscillations using the following function, which retains only the basic oscillatory terms of Eqs. (14) and (15):

$$
\begin{aligned}
\widetilde{S}_{x x}= & C+\left[C^{O} \cos \left(\frac{2 \pi f_{\mathrm{avg}}}{B}-\phi_{\mathrm{avg}}\right)+C^{M} \sin \left(\frac{2 \pi f_{\mathrm{avg}}}{B}\right.\right. \\
& \left.\left.-\phi_{\mathrm{avg}}\right)\right] \cos \left(\frac{2 \pi \Delta f}{B}-\Delta \phi\right),
\end{aligned}
$$

where the phases $\phi_{\text {avg }}$ and $\Delta \phi$ are those determined from fitting $\widetilde{\rho}_{x x}$ at nominally the same temperature, and $C^{O}$ and $C^{M}$ correspond to the amplitudes of the parts in phase and $\pi / 2$ out of phase with $\widetilde{\rho}_{x x}$, i.e., the entropy and Mott components, respectively; $C$ is a simple constant that is close to zero. These amplitudes are, of course, field dependent but for single periods this expression gives excellent fits and gives the average amplitude of the two components at the field corresponding to the center of the oscillation. The results for $C^{O}$ and $C^{M}$ are shown in Fig. 6 for the data at $22.4 \mathrm{~K}$. The amplitudes below about $2.5 \mathrm{~T}$ become unreliable due to noise. The curves through the data points are the best fits using the theoretical expressions for the field dependence of $C^{O}$ and $C^{M}$ from Eqs. (14) and (15), with only the absolute amplitudes left as adjustable constants (see below). These fits to the two components are reasonable indicating that they have been identified correctly. They also show that the Obraztsov (entropy) term dominates at high fields and the Mott term dominates at low fields. This results from the fac- tor $1+\beta^{2} \sim B^{2}$ in the denominator of Eq. (15) and the extra factor of $B$ in the numerator of Eq. (14).

The data at all the other temperatures have been analyzed in the same manner with similar results, but we will not present them here because the signal-to-noise ratio decreases at higher temperatures. Instead, we found it better to utilize all the data at each temperature simultaneously by fitting to the sum of Eqs. (14) and (15), leaving only the individual amplitudes, say $A^{O}$ and $A^{M}$ as adjustable constants. Using the measured value of $f_{\text {avg }}=91.5 \mathrm{~T}$, the expected amplitudes are given by

$$
\begin{aligned}
A^{O}= & -\frac{3 k_{B}}{2|e|}\left(\frac{1}{2 f_{\text {avg }}}\right)^{3 / 2}=-0.052 \mu \mathrm{V} / \mathrm{K} \mathrm{T}^{3 / 2}, \\
A^{M} & =-\frac{\pi k_{B}}{\mu^{2}|e|}\left(1+5 \lambda_{0}^{e} / 2 \lambda^{g}\right)\left(\frac{1}{2 f_{\text {avg }}}\right)^{1 / 2} \\
& =-\left(40 / \mu^{2}\right) \quad \mu \mathrm{V} \mathrm{T}^{3 / 2} / \mathrm{K}
\end{aligned}
$$

with $\lambda_{0}^{e} / \lambda^{g} \approx 0.4$. In the case of $A^{M}, \mu^{2}$ appears in the amplitude because the factor $1+\beta^{2} \approx \beta^{2}=\mu^{2} B^{2}$.

The fits were always excellent and examples are plotted in Fig. 5. The average experimental value we obtain for $A^{O}$ is $(-0.090 \pm 0.008) \mu \mathrm{V} / \mathrm{K} \mathrm{T}^{1 / 2}$, where the uncertainty quoted is only the statistical error. There is no obvious $T$ dependence that might arise if there was a significant admixture of $\widetilde{S}_{x x}^{g}$. There are also uncertainties $\sim 25 \%$ from the probe and thermometer spacing that must be added. However, it seems reasonable that these may be allowed for as follows. We believe that the high field value of $\bar{S}_{x x}=-\pi^{2} k_{B}^{2} T / 2|e| \varepsilon_{F}$ given by either Eq. (12) or Eq. (6) is a reliable prediction. For our sample we expect $-\pi^{2} k_{B}^{2} / 2|e| \varepsilon_{F}=-0.225 \mu \mathrm{V} / \mathrm{K}$. The average experimental value over the range $20-40 \mathrm{~K}$ is $(-0.264 \pm 0.020) \mu \mathrm{V} / \mathrm{K}$ (again with no $T$ dependence). Assuming that $A^{O}$ is incorrect by the same factor gives a corrected $A^{O}=(-0.076 \pm 0.008) \mu \mathrm{V} / \mathrm{K} \mathrm{T}^{3 / 2}$. This amplitude is almost 50\% higher than the expected value. It seems probable that there might still be systematic errors of the order of about $10 \%$ from errors on $T$ and other causes, but the fact that the absolute amplitude of the resistivity oscillations agrees with theory so well suggests that there are no major sources of error. We should mention that if the Dingle factor is omitted when we analyze the data, as in Obraztsov's original result (12), the experimental amplitude agrees well with that predicted. However, the Dingle factor appears to be necessary on physical grounds.

The average experimental value of $A^{M}=(-5.5$ $\pm 1.0) \mu \mathrm{VT}^{3 / 2} / \mathrm{K}$. In this case we might have expected a $T$ dependence from $\mu$ but the scatter on the results appears to be random. We correct this experimental value as we did with $A^{O}$ to give $A^{M}=-4.7 \pm 0.8 \mu \mathrm{V} \mathrm{T}^{3 / 2} / \mathrm{K}$. The measured $\mu$ decreases from 4.6 to $4.0 \mathrm{~m}^{2} / \mathrm{V}$ s over the range $20-40 \mathrm{~K}$, giving a predicted range of $A^{M}=-1.9$ to $-2.5 \mathrm{~m}^{2} / \mathrm{V} \mathrm{s}$. There is a discrepancy of about a factor of 2. Because we have normalized the values to the expected result for the background, we cannot ascribe any remaining error to thermometer or contact spacing. We note, however, that the Mott contribution is small over most of the field range, which 
probably implies that it has larger absolute errors than the entropy term. Nevertheless the discrepancy is certainly real. There are several possible reasons why there could be discrepancies between the experimental and theoretical values of both $A^{M}$ and $A^{O}$.

First, specifically for the case of $A^{M}$, we know from previous work ${ }^{6}$ that $\mu$ obtained from $\rho_{0}$ is too large to obtain good fits to $S_{y x}^{d}$. This seems to be related with the presence of inelastic electron scattering. ${ }^{19}$ It is not a small effect and results in an effective $\mu$ that is $50 \%$ smaller than that measured at $50 \mathrm{~K}$, with smaller differences at lower $T$. Agreement between the experimental and predicted results on $A^{M}$ requires an average $\mu \sim 2.9 \mathrm{~m}^{2} / \mathrm{V} \mathrm{s}$. This is not unreasonable and suggests that this is probably the major source of the discrepancy.

The second, which applies equally to both $A^{M}$ and $A^{O}$, is that the effective mass $m^{*}=0.065 m_{e}$, which we are using for the analysis might be in error. There is a decrease in both $A^{M}$ and $A^{O}$ by about $-6.5 \%$ for each decrease in $m^{*}$ by $0.001 m_{e}$. However, it would also decrease the experimental amplitude of $\widetilde{\rho}_{x x}$ by the same amount and destroy the good agreement with theory that we obtain there.

A final possibility is that Eq. (4) for $\widetilde{\rho}_{y x}$, which is used in Eq. (10), is not correct for $\mathrm{HgSe}: \mathrm{Fe}$. Equations (2) and (4) show that the contributions from $\widetilde{\rho}_{x x}$ and $\widetilde{\rho}_{y x}$ to Eq. (8) are of opposite sign with the latter being $\frac{3}{5}$ times the magnitude of the former. As we have already mentioned, Eq. (4) would be incomplete, or even invalid, if $\varepsilon_{F}$ is pinned so that the number of electrons varies periodically with field. We did, in fact, experimentally investigate $\widetilde{\rho}_{y x}$. As expected the amplitude is very small with $\widetilde{\rho}_{y x} / \bar{\rho}_{y x}<0.001$ for $T>20 \mathrm{~K}$. Unfortunately, we were unable to determine the phase of $\widetilde{\rho}_{y x}$ because different sets of probes yielded slightly different phases, even though the amplitudes were quite reproducible. Presumably this arises from small inhomogeneities in the crystal and the fact that $\rho_{y x}$ cannot not be measured on the probes used for $S_{x x}$ and $\rho_{x x}$. (We also tested the data of Tieke et al. ${ }^{6}$ but these had the same problem). The field dependence of the amplitude could not be satisfactorily fitted to Eq. (4) suggesting that this equation is either invalid or incomplete. There remains a possibility that the actual phase was not the same as $\widetilde{\rho}_{x x}$. If there is a component shifted by $\pm \pi / 2$, there would be a corresponding Mott component of $\widetilde{S}_{x x}^{d}$ indistinguishable from the entropy component and leading to incorrect values for both $A^{M}$ and $A^{O}$. There is some theoretical support for this possibility. When $\hbar \omega_{c} / \epsilon_{F}=B / f \sim 1$ the phase of $\tilde{\rho}_{y x}$ is expected to shift by $\pi / 2$ relative to that of $\tilde{\rho}_{x x} \cdot{ }^{14,15}$ Although this has been seen experimentally, ${ }^{12,23}$ it should be a small effect in the present case where $B / f \leqslant 0.1$. However, a similar phase difference is also expected when the scattering potential is long range, ${ }^{24}$ a situation that might well occur in $\mathrm{HgSeFe}$. Clearly this is a problem that deserves further experimental and theoretical study.

\section{CONCLUSIONS}

This paper has presented experimental data on oscillations in the diffusion thermopower of a degenerate semiconductor, which are essentially free of phonon drag. We have shown that the oscillations have two components, the usual one that can be traced to oscillations in the electronic relaxation time, which we refer to as the Mott contribution, and one originating from the oscillatory magnetization of the sample, which reflects the entropy per unit charge. Due to different field dependences, the former is dominant at low fields and the latter at high fields. The components are separable because they have different phases compared to resistivity oscillations. The measured amplitudes of the two components, especially the entropy term, are higher than expected but all other features are in accord with predictions. In the process of carrying out the above, we also investigated the oscillations in the resistivity. These were found to obey the theoretical predictions in all respects, including their absolute amplitude.

\section{ACKNOWLEDGMENT}

This work was supported by a grant from the Natural Sciences and Engineering Research Council of Canada.
${ }^{1}$ A. F. Ioffe, Semiconductor Thermoelements and Thermoelectric Cooling Infosearch, London, 1956).

${ }^{2}$ Yu. N. Obraztsov, Fiz. Tverdogo Tela 7, 573 (1965) [Sov. Phys. Solid State 7, 455 (1965)].

${ }^{3}$ I. G. Kuleev, Phys. Met. Metallogr. 30, 33 (1970).

${ }^{4}$ M. Jonson and S. M. Girvin, Phys. Rev. B 29, 1939 (1984); H. Oji, ibid. 29, 3148 (1984).

${ }^{5}$ L. Smrčka and P. Streda, J. Phys. C 10, 2153 (1977).

${ }^{6}$ B. Tieke, R. Fletcher, J. C. Maan, W. Dobrowolski, A. Mycielski, and A. Wittlin, Phys. Rev. B 54, 10565 (1996).

${ }^{7}$ A. Miele, R. Fletcher, E. Zaremba, Y. Feng, C. T. Foxon, and J. J. Harris, Phys. Rev. B 58, 13181 (1998).

${ }^{8}$ B. Schroder and G. Landwehr, Solid State Commun. 22, 589 (1977).
${ }^{9}$ I. M. Tsidilkovski and I. G. Kuleyev, Semicond. Sci. Technol. 11, 625 (1996).

${ }^{10}$ I. G. Kuleev, I. I. Lyapinin, A. T. Lonchakov, and I. M. Tsidil'kovskii, Fiz. Tekhn. Poluprovodn. 28, 937 (1994) [Semiconductors 28, 544 (1994)]; I. G. Kuleev, A. T. Lonchakov, I. Yu. Arapova, and G. I. Kuleev, Zh. Eksp. Teor. Fiz. 114, 191 (1998) [JETP 87, 106 (1998)].

${ }^{11}$ E. N. Adams and T. D. Holstein, J. Phys. Chem. Solids 10, 254 (1959); L. M. Roth and P. N. Argyres, in Semiconductors and Semimetals, edited by R. K. Willardson and A. C. Beer (Academic Press, New York, 1966), Vol. 1, p. 159.

${ }^{12}$ R. G. Mani, J. R. Anderson, and W. B. Johnson, in High Magnetic Fields in Semiconductors, edited by G. Landwehr (Springer, Berlin, 1986), p. 436. 
${ }^{13}$ Philip B. Horton, Ph.D. thesis, Louisiana State University, 1964.

${ }^{14}$ G. I. Guseva and P. S. Zyryanov, Phys. Status Solidi 25, 775 (1968).

${ }^{15}$ P. S. Zyryanov and I. G. Kuleyev, Phys. Met. Metallogr. 28, 14 (1969).

${ }^{16}$ M. von Ortenberg, J. Phys. C 21, 5393 (1988); Supercond. Sci. Technol. 8, S16 (1993).

${ }^{17}$ I. G. Kuleev, A. T. Lonchakov, I. I. Lyapilin, and M. Tsidil'kovskii, Zh. Eksp. Teor. Fiz. 103, 1447 (1993) [JETP 76, 707 (1993)].

${ }^{18}$ E. Warekois, M. Lavine, A. Mariano, and H. Gatos, J. Appl. Phys.
33, 690 (1962).

${ }^{19}$ I. M. Tsidilkovsi and I. G. Kuleyev, Supercond. Sci. Technol. 11, 625 (1996).

${ }^{20}$ L. M. Roth, Phys. Rev. 173, 755 (1968).

${ }^{21}$ M. M. Miller and R. Reifenberger, Phys. Rev. B 38, 4120 (1988).

${ }^{22}$ N. G. Gluzman, L. D. Sabirzyanova, I. M. Tsidil'kovskii, L. D. Paranchich, and S. Yu. Paranchich, Fiz. Tekhn. Poluprovodn. 20, 1994 (1986) [Sov. Phys. Semicond. 20, 1251 (1986)].

${ }^{23}$ G. A. Antcliffe and R. A. Stradling, Phys. Lett. 20, 119 (1966).

${ }^{24}$ I. G. Kuleev and S. G. Novokshonov, Fiz. Nizk. Temp. 2, 123 (1976) [Sov. J. Low Temp. Phys. 2, 64 (1976)]. 